\title{
Multi-modality treatment of locally advanced lung cancer: a focus on radiotherapy
}

\author{
Simona Castiglioni, Flora Cammarano, Cinzia Plasmati, Erika Di Betta, Paola Grosso \\ Radiation Oncology Unit, Casa di Cura San Pio X Humanitas, Milan, Italy \\ Contributions: (I) Conception and design: S Castiglioni; (II) Administrative support: S Castiglioni; (III) Provision of study materials or patients: \\ S Castiglioni, F Cammarano, C Plasmati; (IV) Collection and assembly of data: S Castiglioni, E Di Betta, P Grosso; (V) Data analysis and \\ interpretation: S Castiglioni, P Grosso; (VI) Manuscript writing: All authors; (VII) Final approval of manuscript: All authors. \\ Correspondence to: Simona Castiglioni, MD. Radiotherapy Oncology Unit, Casa di Cura San Pio X Humanitas, Milan, Italy. \\ Email: simona.castiglioni@sanpiox.humanitas.it.
}

\begin{abstract}
Lung cancer is the main cause of cancer-related death in the world. Non-small cell lung cancer (NSCLC) accounts for the predominance of lung cancers, in more than half of cases NSCLC' patients are already locally advanced to diagnosis. The therapeutic approach in this stage of disease is complicated and it is therefore essential to manage it in a multidisciplinary context. Surgery remains the therapy of choice, but not all patients have operable disease. For resectable patients, a multimodal approach that involves, in addition to surgery, also systemic and radiation therapy (RT), improves treatment outcomes. The treatment of choice for patients not susceptible to surgery is radiotherapy concomitant with chemotherapy. Implementation in RT techniques, the reduction of the treatment volume with the integration of targeted therapy could lead, in the future, to further improvement in local control (LC) overall survival (OS) results. RT therefore comes into play in almost all locally advanced patients. The aim of this article is to describe the role of RT with curative intent in this setting of patients.
\end{abstract}

Keywords: Radiotherapy (RT); non-small cell lung cancer (NSCLC); locally advanced disease; multi-modality therapy

Received: 31 March 2020; Accepted: 03 November 2020; Published: 25 May 2022.

doi: $10.21037 /$ ccts-20-72

View this article at: http://dx.doi.org/10.21037/ccts-20-72

\section{Introduction}

Non-small cell lung cancer (NSCLC) accounts for about $80 \%$ of all lung cancers, and the therapeutic strategy is linked to the stage of the disease and the general condition of the patient. Stage III disease, according to TumorNode-Metastasis (TNM) Staging System, includes the so-called locally advanced disease and counts about half of NSCLC at the time of diagnosis $(1,2)$. Stage III is in turn divided into IIIA and IIIB which differ for prognosis, treatment options and long-term outcomes. Stage IIIA includes patients with different locally advanced disease, encompassing those with ipsilateral mediastinal lymph node metastases (N2). Optimal management of clinical stage IIIA NSCLC is controversial, being in the "gray zone" between operable (I-II) and inoperable (IIIB-IV) stages. A general schematic management approach is not appropriate, and a multi-modality treatment strategy converging on surgery, radiotherapy (RT) and systemic agents is usually indicated $(3,4)$. Survival is still disappointing despite the progress of new surgical techniques, the introduction and improvement of radiation technologies combined with a greater understanding of disease biology, and tumour progression mechanisms. Surgery remains the treatment of choice for patients diagnosed with operable disease, with the possible integration of an adjuvant or neo-adjuvant treatment to improve treatment outcomes. For patients with resectable locally advanced NSCLC, in case of mediastinal lymph nodes involvement or positive resection margins, there is indication for adjuvant radiotherapy sequentially or concurrently with adjuvant chemotherapy (CT). Selected 
patients, not operable to the diagnosis, can be brought back to the surgery thanks to a neoadjuvant chemo-radiotherapy. For patients with unresectable locally advanced NSCLC, the treatment of choice is concurrent chemo-radiotherapy. An Italian survey focused on therapeutic management of locally advanced NSCLC found that, for stage IIIA/N2single station, $43 \%$ of physicians (radiation oncologists, medical oncologists, thoracic surgeons and pneumologists) proposed neoadjuvant treatment followed by resection, while for stage IIIA/N2-multiple stations $32 \%$ of physicians supported a radical chemoradiation and $23 \%$ proposed surgery after neoadjuvant treatment (5). Therefore, for patients with locally advanced disease, the importance of a multiple therapeutic approach from the time of diagnosis is increasingly evident. Major goals of radiotherapy include local disease control and improvement of the patients' overall prognoses. The purpose of this article is to focus on the role of radiation therapy (RT) in this complicated and heterogeneous kind of patients.

\section{Resectable stage III NSCLC}

\section{Neoadjuvant radiotherapy}

When possible in stage III operable patients, surgical resection remains the treatment of choice but unfortunately the results in terms of overall survival (OS) and local control (LC) remain disappointing. Considering this, in order to improve the percentages of OS and LC, and to improve the radicality rates of surgery, the combination with CT and/ or RT was explored. The idea was that chemotherapy could potentially eradicate micrometastases before resection, reducing tumour size and increasing the probability of resection, while RT played a key role in reducing local tumour recurrence by improving LC. Analysing the trimodal approach, the phase III study INT 0139 conducted by Albain et al., published by Lancet in 2009, randomised 400 potentially operable patients receiving concomitant radio-chemotherapy (45 Gy with cisplatin/etoposide) to surgery versus definitive radiotherapy (60 Gy) and found no significant survival advantage to surgery after chemoRT, despite significantly prolonged progression free survival (PFS) (6). The lack of OS benefit in patients undergoing surgery may relate in large part to the high postoperative death rate following pneumonectomy, predominantly due to acute respiratory distress syndrome (ARDS) and other respiratory causes. Thus, this exploratory analysis could be useful as an adjunct in decision-making, to raise a caution flag when considering a trimodality prescription with pneumonectomy. Therefore, the trimodality approach could be superior if lobectomies are performed. A European randomized multi-centered trial investigated whether the addition of neoadjuvant radiotherapy to neoadjuvant chemotherapy prior to surgical resection improves outcomes (7). In this trial, the addition of preoperative RT was feasible without increasing surgical complications, even in patients (23\%) who underwent pneumonectomy. While preoperative chemoradiation did not improve survival, patients who received RT had more frequent pathologically complete response, complete resection, and mediastinal downstaging, which all indicate local efficacy of RT. The authors therefore suggested that the use of one local treatment, RT or surgery, in combination with CT, should be considered the standard treatment. EORTCLCG trial randomly assigned stage IIIA-N2 patients responding to induction chemotherapy to surgical resection or radiotherapy (8). The data reported that neither surgery nor radiotherapy demonstrated superiority in terms of OS or PFS. The authors concluded that radiotherapy should be considered the locoregional therapy of choice to combine with CT for these patients, given the lower invasiveness and the lower risk of mortality compared to surgery. This study has been criticized because only $50 \%$ of resected diseases were pathologically radical (R0) and because $47 \%$ of surgical procedures were pneumonectomies. A randomized phase III trial, comparing surgery to radical radiotherapy after induction CT, demonstrated a comparable 5-year OS and PFS rates in both groups of patients, and concluded that radical chemoradiation was comparable with neoadjuvant CT and surgery (9). These data have been confirmed by a meta-analysis published in 2018 specifically including only randomized control trials of patients diagnosed exclusively with stage IIIA/N2 NSCLC (10). The results show that induction chemoradiotherapy, compared to induction chemotherapy alone, is associated with a greater tumour response and mediastinal downstaging in patients with resectable stage IIIA NSCLC, without increasing periinterventional mortality. However, it does not improve long-term survival.

In conclusion, neoadjuvant chemo-radiotherapy has proven to get downstaging of mediastinal nodal disease and to improve complete resection, and thus should be considered for selected patients.

\section{Post-operative radiotherapy (PORT)}

In resected locally advanced NSCLC patients, administration 
of adjuvant chemotherapy was considered the standard of care (11). However, up to $40 \%$ of resected patients, despite having complete resection and adjuvant CT, undergo local relapse. With the purpose of improving LC and OS, PORT was evaluated in historical randomized control trials $(12,13)$, the results of which revealed the difficulty of defining ideal patients to candidate for PORT. PORT meta-analysis of all available randomised trials (nine trials including 2,128 patients) was conducted to provide a guidance for clinical practice based on observed results (14). For OS there was evidence of a detrimental effect by PORT, with a $21 \%$ relative increase in the risk of death resulting in an overall reduction in survival from $55 \%$ to $48 \%$ at 2 years. Analysing the data by stage and by nodal status, however, in stage III/N2 patients the authors found an improvement in OS although not significant, while in stage I-II/N0-N1 patients confirmed a detriment likely due to excess of toxicity. This meta-analysis was criticised due to the population in question: the patients analyzed were part of studies conducted in years in which radiotherapy techniques were much more limited than the current ones, and therefore potentially burdened with greater toxicity. It cannot be excluded that RT modern techniques, with reduced cardiac and pulmonary toxicity, could lead to a more accurate assessment of role of PORT. Analysis of 202 patients treated with surgery and modern-technique PORT did not find an increase in the risk of intercurrent deaths (15). More recently, a retrospective analysis of the SEER database analyzed 7,465 stage II-III patients treated in 1988-2002, therefore irradiated with more modern RT techniques than patients evaluated in the previously mentioned metaanalysis (16). The authors concluded that PORT resulted in improved survival in $\mathrm{N} 2$ patients but worsened it in N0-N1 patients. Given the heterogeneity of stage III/N2 patients, more specific factors such as the number of N2 metastases and/or N2 stations were evaluated. A recent meta-analysis supports the role of PORT in $\mathrm{pN} 2$ patients with multiple $\mathrm{N} 2$ metastases, not finding significant difference in OS for patients with single N2 station involvement (17). The ongoing LungART trial is assessing the role of PORT with modern RT techniques.

\section{Non-resectable stage III NSCLC}

\section{Multimodality strategy}

In stage III NSCLC not amenable to curative resection, historical randomized trials have been designed to define the survival advantage when chemotherapy was associated with
RT compared with exclusive RT (18-20). RT provides LC and chemotherapy not only prevents or reduces the potential micro-metastases but also enhances RT efficacy with a radiosensitivity mechanism. The results of two important meta-analysis demonstrated that patients with locally advanced NSCLC who underwent RT with radical doses, also treated with sequential or concomitant chemotherapy, showed an improvement in survival $(21,22)$. Data analysis showed an absolute survival benefit of $2.2 \%$ at 5 years when combining chemotherapy wit RT, compared to exclusive RT. Standard RT dose was typically 60-66 Gy in 2 Gy daily fractions with platinum-based doublet chemotherapy. However, the association of treatments was not bound by timing, which could be sequential or concomitant. Later randomized trials aimed to evaluate the impact of timing (concomitant versus sequential) of radio-chemotherapy on outcomes in survival and disease control $(23,24)$. Aupérin et al., in a meta-analysis of six randomized trials published on 7 Clin Oncol in 2010, demonstrated a significant 16\% relative reduction in mortality with the concomitant scheduling, with a survival rate of $18.4 \%$ at 3 years and of $15.1 \%$ at 5 years (23). There was no difference in distant failure rates between the two treatments schedules therefore it is arguable to think that the highlighted improvement is attributable to a decrease in the risk of loco-regional relapse. The authors found no differences in the results depending on the number of chemotherapy used. Concomitant CT regimen with two or three agents had better PFS than single agents, but no difference was found in OS. The improvement of loco-regional control with the concomitant treatment confirmed the well-known radiosensitizing effect linked to platinum-based chemotherapy. This radiosensitizing effect also had an impact on healthy organs, with evidence of greater acute esophageal toxicity in patients treated with concomitant rather than sequential combination. As regards acute pulmonary toxicity, however, there was no difference between the two groups. In the randomized phase III trial RTOG 9410 610, patients with inoperable stage III NSCLC were randomly assigned to concomitant or sequential chemo-radiotherapy (CT-RT) (24). OS was significantly higher for patients treated with concomitant therapy, even though the acute toxic effects rate (esophagitis) were higher than in the sequential group. The limitation of this study was that the majority of patients had a good performance status and/or other comorbidity conditions. Patients with lower functional status probably would have tolerated worse the severe esophagitis observed with concurrent therapy. Therefore, performance status and the 
presence of any comorbidity must be considered to define the feasibility of concomitant treatment. An option for frail patients could be concomitant CT-RT with daily low-dose cisplatin, with lower risk of hematologic and cardiac toxicity, or sequential CT-RT (25). Age is also a pivotal factor, as it was proven by a retrospective study which reviewed 130 patients over 70 years with locally advanced NSCLC who did not undergo surgery (26). The study showed that the use of combined treatment modality was associated with better survival, and with the greatest survival observed in patients treated with concurrent CT-RT. The authors therefore suggested that concomitant CT-RT, when feasible, should be strongly recommended also in patients 70 years or more. New anticancer agents such as targeting drugs combined with RT could lead, in the future, towards treatments with an increasingly favorable therapeutic index (27-29). Research in recent years has led to the introduction into clinical practice of several novel agents, including immune checkpoint inhibitors that specifically target PD-L1 of the immune system pathway. The introduction of these agents as consolidation therapy after CT-RT could represent the turning point in the prognosis of these patients. In NSCLC, from the radiobiological point of view, RT induces an immunological modification in the local tumor microenvironment which, thanks to a synergistic effect, could lead to enhanced responses by increasing the exposure to immune system cells. In a study of patients with advanced NSCLC treated with pembrolizumab, PFS and OS were longer in those who had previously received RT than in those who had not (30). A randomized phase II trial aimed at evaluating the impact of stereotactic body radiation therapy (SBRT) in patients with single metastasis before receiving pembrolizumab: the irradiated patients experienced an improved response rate (41\% vs. $19 \%)$ and PFS (6.4 vs. 1.8 months) (31). It could be argued that there is an overlapping toxicity such as pneumonitis, but the optimal RT dose and volume are still under evaluation in order to reach a better synergism without severe toxicity.

\section{Radiotherapy technique and dose}

The critical aim of modern RT technique is to maximize tumour control and to minimize treatment toxicity. Considerable effort has been directed at attempting to optimise the use of radiotherapy. The technological implementation, with improvement of treatment technique and its verification systems, leads to new research developments in the field of dose fractionation and dose escalation.

\section{Dose and fractionation}

According to the radiobiological model, the way to improve LC and OS includes dose escalation and this was associated with better outcomes also in retrospective analysis for NSCLC' patients (32). The phase III study RTOG 0617 evaluated the role of RT dose escalation in patients with unresectable stage IIIA/B NSCLC: patients were randomly assigned to receive, with concomitant chemotherapy, RT with standard dose to 60 Gy or dose escalation to 74 Gy (33). The median OS was 28.7 months for $60 \mathrm{~Gy}$ arm and 20.3 months for 74 Gy arm $(\mathrm{P}=0.004)$. Although the objective of the study was to demonstrate an advantage for patients treated with a higher dose, the results showed the opposite: survival in patients treated with higher doses (74 Gy) was significantly lower than in patients treated with conventional doses (60 Gy). The explanation could be a higher incidence of treatment-related deaths in the high-dose group associated to heart radiotoxicity. It has indeed been proven that factors predictive of better OS on multivariable analysis included tumor location away from the heart, smaller RT tumor volume and reduction of the heart dose as much as possible. In addition, analysing acute toxicity, dose escalation was associated with more severe esophagitis than standard dose. As a conclusion, the authors recommended maintaining the conventional dose of $60 \mathrm{~Gy}$ in the setting of concurrent CT-RT. Accelerated hyper-fractionation is a RT modality in which the radiant treatment is performed with a lower dose per session but more than one time a day. Several randomized trials have been conducted to evaluate the benefit of this fractionation over conventional RT. A meta-analysis that evaluated these randomized trials showed a modest benefit, not statistically significant, in terms of OS of the hyper-fractioned scheme compared to the conventional scheme (34). Moreover, hyper-fractionation did not significantly improve PFS and demonstrated a greater toxicity, particularly severe esophagitis, compared to conventionally fractionated RT. SBRT is hypofractionation RT which delivers a higher dose per fraction compared to conventional fractionated schedules, with a potential higher BED to provide better LC. Phase I studies have recently been published showing the feasibility and safety of dose-escalated SBRT boost after concomitant CT-RT $(35,36)$. To better define the residual area of disease where to perform the SBRT, functional information can be particularly useful. We are waiting for 
the results of a phase II study (RTOG 1106), evaluating an individualized adaptive RT plan compared with standard RT; in the study, a mid-treatment PET/CT has made to allow a hypofractionated boost to escalate total dose to uptake areas.

\section{Technology}

The introduction of new and advanced technologies in imaging and techniques has improved the accuracy of RT, with better tumor coverage and low dose to organs at risk. Systematic integration of three-dimensional conformal radiotherapy (3D-CRT) has undoubtedly contributed to improving the results. Intensity-modulated radiation therapy (IMRT) delivered even more conformal treatment, with multiple beams that during the treatment modify the RT fluence and shape. Imaging capabilities have progressed and cone-beam CT (CBCT) has replaced traditional imaging to ensure accurate set-up. Although RT dose and volume are standardized, technological advances allow individualized RT plans. This technological implementation, with an optimal treatment plan through more compliant dose distributions and a better definition of the target volume, has allowed a safer dose escalation in locally advanced NSCLC with reduction of toxicity (37-40). IMRT is a type of conformal RT with a computer-aided optimization process to deliver higher dose to tumor volume, while better sparing the surrounding normal structure. The IMRT problem is the possible exposition of large volume of lungs to low-doses of RT, with potential increased risk of pulmonary toxicity and secondary malignancy. Nevertheless, non-randomized studies that have explored the use of advanced technologies demonstrated reduced toxicity and improved OS versus older techniques. In a secondary analysis of the RTOG 0617 trial, IMRT was associated with lower rates of high-grade radiation pneumonitis (from $7.9 \%$ to $3.5 \%$ ) and with lower cardiac doses (which is an important predictor of survival) compared to 3D-CRT $(41,42)$. Multivariable analysis in NRG Oncology RTOG 0617 experience showed that the position of the tumor away from the heart was a predictive factor of better OS (41). The results are even more significant because the authors confirmed that, despite being used to treat larger and more aggressive tumors, IMRT was associated with a reduced risk of lung toxicity. Therefore, in patients with advanced lung cancer, techniques as IMRT or volumetric modulated arc therapy (VMAT) are appropriate and highly recommended to improve target dose conformity and organ-at-risk (OAR) sparing (38). A retrospective study reviewed 652 locallyadvanced NSCLC patients, including 206 treated with 3D-CRT and 446 with IMRT (43). The authors found that patients receiving IMRT gained a significantly improved local-regional PFS compared with 3D-CRT. Subgroup analyses indicated that patients who were female, nonsmokers, with pathological adenocarcinoma, also showed a benefit in term of OS and distant metastasis-free survival. Further, the use of IMRT was correlated with reduction in radiation-induced lung toxicity and esophageal toxicity. RT volume too has favorably impacted toxicity. Elective nodal irradiation, which was systematically used in the past, is not currently part of the routine, as the probability of lymph node recurrence in elective sites is less than $10 \%(44,45)$. In more recent trials esophageal toxicity has decreased considerably, restricting the RT treatment volume to the tumor and involved nodes only (44). In this scenario, some committees, such as EORTC, provided recommendations for using the most appropriate technology for the best clinical practice and for prospective studies in radiation treatment $(45,46)$.

\section{Conclusions}

Locally advanced NSCLC requires multimodal treatment in which RT plays an important role on several fronts. Numerous data demonstrated that relapses occur in the majority of patients both locally and at distant sites. It has been largely shown that the volume and extent of the tumour with the involvement of the lymph nodes determines overall survival. Therefore, optimal LC as well as systemic treatment are essential. Several studies show us that patients have a significant risk of failure not only for distant metastases but also for loco-regional relapse. Thus, it is important for the future to redefine the impact of dose escalation and different fractionation with the use of cuttingedge technology that minimizes toxicity. This may affect prognosis, as it is increasingly evident that $\mathrm{RT}$ can favorably contribute to systemic control. Radiations act at the level of the tumor microenvironment with immunomodulatory effects also outside the treated field. In the future, this could be the rationale for synergistic combination approach with immunotherapy, which could complement the established role of the combined RT-CT treatment. In this scenario, with more advanced technologies and the synergy with medical therapies, the future could lead to increasingly personalized RT treatments with greater efficacy and less toxicity. 


\section{Acknowledgments}

Funding: None.

\section{Footnote}

Provenance and Peer Review: This article was commissioned by the Guest Editors (Davide Tosi and Alessandro Palleschi) for the series "The Treatment of Locally Advanced Lung Cancer" published in Current Challenges in Thoracic Surgery. The article has undergone external peer review.

Conflicts of Interest: All authors have completed the ICMJE uniform disclosure form (available at https://ccts.amegroups. com/article/view/10.21037/ccts-20-72/coif). The series "The Treatment of Locally Advanced Lung Cancer" was commissioned by the editorial office without any funding or sponsorship. The authors have no other conflicts of interest to declare.

Ethical Statement: The authors are accountable for all aspects of this work in ensuring that questions related to the accuracy or integrity of any part of this work are appropriately investigated and resolved.

Open Access Statement: This is an Open Access article distributed in accordance with the Creative Commons Attribution-NonCommercial-NoDerivs 4.0 International License (CC BY-NC-ND 4.0), which permits the noncommercial replication and distribution of the article with the strict proviso that no changes or edits are made and the original work is properly cited (including links to both the formal publication through the relevant DOI and the license). See: https://creativecommons.org/licenses/by-nc-nd/4.0/.

\section{References}

1. NCCN Clinical Practice Guidelines in Oncology: nonsmall cell lung cancer. 2020 (3.2020). Available online: http://www.nccn.org/professionals/physician_gls/pdf/ nscl.pdf

2. Amin MB, Gress DM, Meyer Vega LR, et al. AJCC Cancer Staging Manual (eight edition). Philadelphia, PA: Springer, 2018.

3. Robinson LA, Ruckdeschel JC, Wagner H, et al. Treatment of non-small cell lung cancer-stage IIIA. ACCP evidence-based clinical practice guidelines ( 2 nd edition). Chest 2007;132:243S-265S.
4. Santana-Davila R, Martins R. Treatment of stage IIIA nonsmall-cell lung cancer: a concise review for the practicing oncologist. J Oncol Pract 2016;12:601-6.

5. Bruni A, Giaj-Levra N, Ciammella P, et al. Management of locally advanced non-small cell lung cancer in the moder era: a national Italian survey on diagnosis, treatment and multidisciplinary approach. PLoS One 2019;14:e0224027.

6. Albain KS, Swann RS, Rusch VW, et al. Radiotherapy plus chemotherapy with or without surgical resection for stage III non-small-cell lung cancer: a phase III randomised controlled trial. Lancet 2009;374:379-86.

7. Pless M, Stupp R, Ris HB, et al. Induction chemoradiation in stage IIIA/N2 non-small-cell lung cancer: a phase 3 randomised trial. Lancet 2015;386:1049-56.

8. van Meerbeeck JP, Kramer GW, Van Schil PE, et al. Randomized controlled trial of resection versus radiotherapy after induction chemotherapy in stage IIIA-N2 non-small-cell lung cancer. J Natl Cancer Inst 2007;99:442-50.

9. Eberhardt W, Gauler T, Pottgen C, et al. Phase III study of surgery $(\mathrm{S})$ versus definitive concurrent chemoradiotherapy boost (def ccCRTx-Box) in patients (pts) with operable $(\mathrm{OP}+)$ stage IIIA(N2)/selected IIIB (sel IIIB) non-small cell lung cancer (NSCLC) following induction (IND) chemotherapy (CTx) and concurrent CRTx (ESPATUE). J Clin Oncol 2014;32:abstr 7510.

10. Chen Y, Peng X, Zhou Y, et al. Comparing the benefits of chemoradiotherapy and chemotherapy for resectable stage III A/N2 non-small cell lung cancer: a meta-analysis. World J Surg Oncol 2018;16:8.

11. Douillard JY, Rosell R, De Lena M, et al. Adjuvant vinorelbine plus cisplatin versus observation in patients with completely resected stage IB-IIIA non-small-cell lung cancer (Adjuvant Navelbine International Trialist Association [ANITA]): a randomised controlled trial. Lancet Oncol 2006;7:719-27.

12. Stephens RJ, Girling DJ, Bleehen NM, et al. The role of post-operative radiotherapy in non-small cell lung cancer: a multicentre randomised trial in patients with pathologically staged T1-2, N1-2, M0 disease. Br J Cancer 1996;74:632-9.

13. Debevec M, Bitenc M, Vidmar S, et al. Post-operative radiotherapy for radically resected N2 non-small cell lung cancer: randomised clinical study 1988-92. Lung Cancer 1996;14:99-107.

14. Postoperative radiotherapy in non-small-cell lung cancer: systematic review and meta-analysis of individual patient data from nine randomised controlled trials. PORT Meta- 
analysis Trialists Group. Lancet 1998;352:257-63.

15. Machtay M, Lee JH, Shrager JB, et al. Risk of death from intercurrent disease is not excessively increased by modern postoperative radiotherapy for high-risk resected nonsmall-cell lung carcinoma. J Clin Oncol 2001;19:3912-7.

16. Lally BE, Zelterman D, Colasanto JM, et al. Postoperative radiotherapy for stage II or III non-small-cell lung cancer using the surveillance, epidemiology, and end results database. J Clin Oncol 2006;24:2998-3006.

17. Liu T, Mu Y, Dang J, et al. The role of postoperative radiotherapy for completely resected pIIIA-N2 non-small cell lung cancer patients with different clinicopathological features: a systemic review and meta-analysis. J Cancer 2019;10:3941-9.

18. Dillman RO, Seagren SL, Propert KJ, et al. A randomized trial of induction chemotherapy plus high-dose radiation versus radiation alone in stage III non-small-cell lung cancer. N Engl J Med 1990;323:940-5.

19. Schaake-Koning C, van den Bogaert W, Dalesio O, et al. Effects of concomitant Cisplatin and radiotherapy on inoperable non-small-cell lung cancer. N Engl J Med 1992;326:524-30.

20. Cakir S, Egehan I. A randomised clinical trial of radiotherapy plus Cisplatin versus radiotherapy alone in stage III non-small cell lung cancer. Lung Cancer 2004;43:309-16.

21. Chemotherapy in non-small cell lung cancer: a metaanalysis using updated data on individual patients from 52 randomised clinical trials. Non-small Cell Lung Cancer Collaborative Group. BMJ 1995;311:899-909.

22. Aupérin A, Le Pechoux C, Pignon JP, et al. On behalf of the Meta-Analysis of Cisplatin/carboplatin based Concomitant Chemotherapy in non-small cell Lung Cancer (MAC3-LC) Group: concomitant radiochemotherapy based on platin compounds in patients with locally advanced non-small cell lung cancer (NSCLC): a meta-analysis of individual data from 1764 patients. Ann Oncol 2006;17:473-83.

23. Aupérin A, Le Pechoux C, Rolland E, et al. Meta-analysis of concomitant versus sequential radiochemotherapy in locally advanced non-small-cell lung cancer. J Clin Oncol 2010;28:2181-90.

24. Curran WJ, Paulus R, Langer CJ, et al. Sequential vs concurrent chemoradiation for stage III non-small cell lung cancer: randomized phase III trial RTOG 9410. J Natl Cancer Inst 2011;103:1452-60.

25. Belderbos J, Uitterhoeve L, van Zandwijk N, et al. On behalf of the EORTC LCG and RT Group: randomised trial of sequential versus concurrent chemo-radiotherapy in patients with inoperable non-small cell lung cancer (EORTC 08972-22973). Eur J Cancer 2007;43:114-21.

26. Sakin A, Sahin S, Atci MM, et al. The effect of different treatment modalities on survival in elderly patients with locally advanced non-small cell lung cancer. Pulmonology 2021;27:26-34.

27. Ko EC, Raben D, Formenti S. The integration of radiotherapy with immunotherapy for the treatment of non-small cell lung cancer. Clin Cancer Res 2018;24:5792-806.

28. Horinouch H. Precision radiotherapy for patients with locally advanced non-small cell lung cancer in the era of immunotherapy and precision medicine. Transl Lung Cancer Res 2018;7:S146-S148.

29. Simone CB 2nd, Burri SH, Heinzerling JH. Novel radiotherapy approaches for lung cancer: combining radiation therapy with targeted and immunotherapies. Transl Lung Cancer Res 2015;4:545-52.

30. Shaverdian N, Lisberg AE, Bornazyan K, et al. Previous radiotherapy and the clinical activity and toxicity of pembrolizumab in the treatment of non-small-cell lung cancer: a secondary analysis of the KEYNOTE-001 phase 1 trial. Lancet Oncol 2017;18:895-903.

31. Theelen W, Peulen H, Lalezari F, et al. Randomized phase II study of pembrolizumab after stereotactic body radiotherapy (SBRT) versus pembrolizumab alone in patients with advanced non-small cell lung cancer: The PEMBRO-RT study. J Clin Oncol 2018;36:9023.

32. Machtay M, Bae K, Movsas B, et al. Higher biologically effective dose of radiotherapy is associated with improved outcomes for locally advanced non-small cell lung carcinoma treated witch chemoradiation: an analysis of Radiation Therapy Oncology Group. Int J Radiat Oncol Biol Phys 2012;82:425-34.

33. Bradley JD, Hu C, Komaki RR. Long-term results of NRG oncology RTOG 0617: standard- versus highdose chemoradiotherapy with or without Cetuximab for unresectable stage III non-small-cell lung cancer. J Clin Oncol 2020;38:706-14.

34. Mauguen A, Le Pechoux C, Saunders MI, et al. Hyperfractionated or accelerated radiotherapy in lung cancer: an individual patient data meta-analysis. J Clin Oncol 2012;30:2788-97.

35. Higgins KA, Pillai RN, Chen Z, et al. Concomitant chemotherapy and radiotherapy with SBRT boost for unresectable, stage III non-small cell lung cancer: a phase I study. J Thorac Oncol 2017;12:1687-95. 
36. Hepel JT, Lenoard KL, Safran H, et al. Stereotactic body radiation therapy boost after concurrent chemoradiation for locally advanced non-small cell lung cancer: a phase I dose escalation study. Int J Radiat Oncol Biol Phys 2016;96:1021-7.

37. Liao ZX, Komaki RR, Thames HD, et al. Influence of technologic advances on outcomes in patients with unresectable, locally advanced non-small-cell lung cancer receiving concomitant chemoradiotherapy. Int J Radiat Oncol Biol Phys 2010;76:775-81.

38. Choi JH. Comparison of IMRT and VMAT plan for advanced stage non-small cell lung cancer treatment. Arch Can Res 2018;19:6-4.

39. Wijsman R, Dankers F, Troost EGC, et al. Comparison of toxicity and outcome in advanced stage non-small cell lung cancer patients treated with intensity-modulated (chemo-) radiotherapy using IMRT or VMAT. Radiother Oncol 2017;122:295-9.

40. Selek U, Bolukbasi Y, Welsh JW, et al. Intensitymodulated radiotherapy versus 3-Dimensional conformal radiotherapy strategies for locally advanced non-small-cell lung cancer. Balkan Med J 2014;31:286-94.

41. Rades D, Glatzel E, Werner EM, et al. Prevalence and characteristics of symptomatic pneumonitis after radiotherapy of patients with locally advanced lung cancer.

doi: $10.21037 /$ ccts-20-72

Cite this article as: Castiglioni S, Cammarano F, Plasmati C, Di Betta E, Grosso P. Multi-modality treatment of locally advanced lung cancer: a focus on radiotherapy. Curr Chall Thorac Surg 2022;4:19.
Anticancer Res 2019;39:6909-13.

42. Chun SG, Hu C, Choy H, et al. Impact of Intensitymodulated radiation therapy technique for locally advanced non-small-cell lung cancer: a secondary analysis of the NRG Oncology RTOG 0617 randomized clinical trial. J Clin Oncol 2017;35:56-62.

43. Wang J, Zhou Z, Liang J, et al. Intensity-modulated radiation therapy may improve local-regional tumor control for locally advanced non-small cell lung cancer compared with three-dimensional conformal radiation therapy. Oncologist 2016;21:1530-7.

44. Yuan S, Sun X, Li M, et al. A randomized study of Involved-Field Irradiation versus Elective Nodal Irradiation in combination with concurrent chemotherapy for inoperable stage III nonsmall cell lung cancer. Am J Clin Oncol 2007;30:239-44.

45. De Ruysscher D, Faivre-Finn C, Moeller D, et al. European Organization for Research and Treatment of Cancer (EORTC) recommendations for planning and delivery of high-dose, high precision radiotherapy for lung cancer. Radiother Oncol 2017;124:1-10.

46. Senan S, De Ruysscher D, Giraud P, et al. Literature-based recommendations for treatment planning and execution in high-dose radiotherapy for lung cancer. Radiother Oncol 2004;71:139-46. 